\title{
Tempestività di intervento nella colica renale: aspetti fisiopatologici
}

Giornale di Tecniche Nefrologiche e Dialitiche 2018, Vol. 30(2) 92-99

(C) The Author(s) 2018

Article reuse guidelines:

sagepub.com/journals-permissions

DOI: $10.1177 / 0394936218760827$

journals.sagepub.com/home/gtn

(S)AGE

\author{
Alberto Trinchieri' e Emanuele Montanari²
}

\begin{abstract}
Timeliness of intervention in the renal colic: physiopathological aspects

Renal colic is one of the most common diagnoses of emergency room access and ureteral stone is one of the most common causes of renal colic due to ureteral obstruction. Ureteral obstruction initially causes an increase of intraureteral pressure, which returns to baseline values after a short time after the reduction in urine production and the progressive increase in the circumference and ureter length. Reduction in urinary flow depends on the renal hemodynamic response, which has three phases: initial vasodilation of the preglomerular afferent artery; vasoconstriction of the postglomerular efferent artery; and, finally, vasoconstriction of the preglomerular afferent artery. Renal parenchyma ischemia is associated with oxidative stress, inflammatory infiltrate and subsequent apoptosis of tubular cells and interstitial fibrosis. This degenerative pathological process tends to continue even after relatively early removal of the obstruction and despite the restoration of renal hemodynamics. Fibrosis progression is associated with alterations in renal function with hyperfiltration in the surviving nephronic population and alterations in tubular function. These pathophysiological aspects have to be considered in the management of patients with renal colic and ureteral obstruction. Expulsive treatment of renal stones should be carefully planned by accurate selection of patients and frequent follow-up controls. When the obstruction is severe or the expulsion time is prolonged, early treatment or temporary urinary diversion with deferred treatment should be considered. The time limit for awaiting spontaneous stone passage is not well defined and varies depending on the degree of obstruction and possible complicating factors, however it may be prudent to not exceed two to three weeks.
\end{abstract}

\section{Keywords}

Renal colic, ureteral stones, unilateral ureteral obstruction, expulsive treatment

\section{Introduzione}

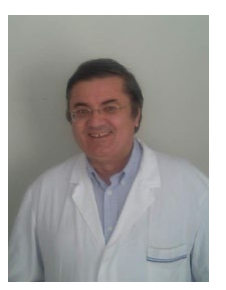

La colica renale è una sindrome dolorosa associata ad ostruzione ureterale intrinseca o estrinseca (tabella 1) dove le calcolosi urinaria è la causa più frequente di ostruzione ureterale.

La colica renale è una delle più comuni diagnosi di accesso al Pronto Soccorso, pari a circa il $30 \%$ di tutti i casi di dolore addominale acuto, che costituiscono circa il 5-10\% di tutte le diagnosi di accesso osservate in ampie casistiche. ${ }^{1}$ In Italia l'incidenza annuale di coliche renali che hanno richiesto l'accesso al Pronto Soccorso è stata stimata nello $0.158 \%$ della popolazione generale. ${ }^{2}$

Il trattamento analgesico ideale per la colica renale non è stato tuttora definito in modo conclusivo. Una prima metanalisi del 2005 non è stata in grado di definire la superiorità del trattamento con infiammatori non steroidei
(FANS) rispetto a quello con oppiacei, sebbene sia stata sottolineata la maggior incidenza di effetti collaterali gastrointestinali degli oppiacei, in particolare della petidina. ${ }^{3}$ Una seconda metanalisi del 2015 è stata limitata alla valutazione dell'efficacia di FANS e di altri farmaci nonFANS e non oppiacei (ad es fluoroglucinolo, paracetamolo). ${ }^{4}$ Nonostante le evidenze disponibili non fossero di elevata qualità è stata confermata l'efficacia dei FANS rispetto agli anticolinergici e al placebo in assenza di effetti collaterali

\footnotetext{
ISC Urologia, Ospedale A. Manzoni, Lecco, Italia

Clinica Urologica, IRCCS Ca' Granda Ospedale Maggiore Policlinico

- Università di Milano, Milano, Italia

Corrispondenza:

Alberto Trinchieri, SC Urologia, Ospedale A. Manzoni, via dell'Eremo 9/II, Lecco 23900, Italia.

E-mail: a.trinchieri@asst-lecco.it
} 
Tabella I. Cause di ostruzione ureterale.

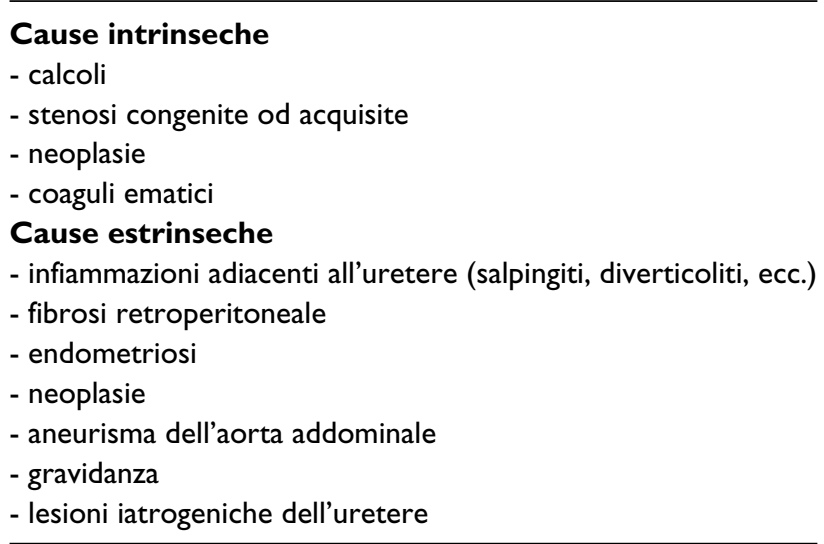

gravi. I dati sui farmaci non-FANS e non-oppiacei non erano sufficienti per una valutazione. A causa di questa persistente incertezza è stata annunciata una nuova metanalisi che includerà alcuni più recenti studi di buona qualità. ${ }^{5} \mathrm{Al}$ momento le linee-guida della European Association of Urology consigliano l'impiego dei FANS come trattamento di prima linea della colica renale. ${ }^{6}$

\section{Aspetti fisiopatologici}

L'ostruzione ureterale implica una serie di alterazioni fisiopatologiche relative alla peristalsi ureterale, al flusso urinario, all'emodinamica renale e all'infiammazione dell'interstizio renale che possono essere all'origine di alterazioni transitorie o permanenti della funzione renale.

Gli effetti dell'ostruzione dipendono dal grado e dalla durata, dalla presenza di fattori complicanti, in particolare dall'infezione, e dalla presenza e dalle condizioni del rene controlaterale.

\section{Fisiologia dell'uretere}

La funzione dell'uretere è di assicurare il trasporto dell'urina dalla pelvi renale alla vescica attraverso il meccanismo peristaltico. La peristalsi ureterale può essere descritta come un' "onda" di contrazione del sincizio muscolare della parete del viscere che lo percorre nella sua lunghezza a partire dalla pelvi renale, ove risiedono le cellule pacemaker che rispondono alla pressione intrapielica, fino alla vescica. ${ }^{7}$ Le cellule pacemaker della pelvi sono differenti dalle altre cellule muscolari lisce dell'uretere in quanto le loro membrane si depolarizzano e ripolarizzano ritmicamente, trasmettendo la depolarizzazione attraverso le gap junctions alle cellule muscolari lisce non-pacemaker dell'uretere che si contraggono per l'effetto dell'afflusso di calcio nel citosol. Le cellule pacemaker inoltre interagiscono con cellule interstiziali Cajalsimili che hanno un ruolo non ancora ben precisato sul meccanismo della peristalsi. Questa ritmicità della contrazione su base miogenica persiste anche dopo interruzione dell'innervazione come accade nell'uretere del rene trapiantato. L'innervazione dell'uretere ha quindi un'azione modulatoria della contrattilità miogenica ed è costituita da un'innervazione autonomica simpatica e parasimpatica, cui si aggiunge l'azione di neurotrasmettitori nonadrenergici noncolinergici. Il sistema simpatico ha un ruolo preminente, coi recettori adrenergici alfa che promuovono la contrazione e quelli beta il rilassamento delle fibre muscolari lisce. I recettori alfa sono più rappresentati e gli alfal bloccanti inibiscono in vitro la contrazione spontanea di segmenti di uretere umano e sono impiegati clinicamente per favorire il passaggio di calcoli nell'uretere. I recettori beta2 e beta3 sono predominanti nel favorire il rilassamento delle cellule muscolari.

In condizioni normali le contrazioni hanno un ritmo lento di due a sei contrazioni per minuto con un aumento della pressione luminale durante la contrazione da valori di riposo di 0-5 $\mathrm{cm} \mathrm{H}_{2} \mathrm{O}$ a 20-80 $\mathrm{cm} \mathrm{H}_{2} \mathrm{O}$. Le contrazioni dell'uretere producono la formazione di boli di urina che percorrono l'uretere, il quale progressivamente aumenta di diametro per poi collabire al termine del passaggio dell'onda di peristalsi. Quando la diuresi aumenta le contrazioni dell'uretere aumentano per frequenza ed ampiezza, fino alla scomparsa dell'intervallo tra un bolo e l'altro.

\section{Fisiopatologia dell'uretere ostruito}

L'ostruzione ureterale causa un aumento di ampiezza della contrazione peristaltica con incapacità dell'uretere a collabire tra le contrazioni. ${ }^{8}$ Le modificazioni anatomofisiologiche dell'uretere successive all'ostruzione sono state ben studiate negli anni settanta in una serie di studi sperimentali nel coniglio. $^{9-14}$ Per descrivere le caratteristiche della contrazione dell'uretere devono essere considerati parametri come la tensione della parete ureterale, che è la misura della forza che tende a far collabire il lume dell'uretere, e la pressione endoluminale dell'uretere, che tende a far progredire l'urina lungo l'uretere in senso distale verso la vescica. L'ostruzione ureterale prolungata comporta una dilatazione ed un allungamento del viscere con conseguente tortuosità dello stesso in associazione a valori di pressione intraluminali relativamente basse.

\section{Fase iniziale}

Immediatamente dopo l'inizio dell'ostruzione si osserva un iniziale incremento della pressione intraluminale "a riposo" dell'uretere e un iniziale aumento delle dimensioni del viscere, sia del diametro che della lunghezza. L'incremento pressorio è dovuto al temporaneo persistere della produzione di urina che si accumula nel tratto a monte dell'ostruzione e provoca, a sua volta, l'aumento di dimensioni dell'uretere, il quale deve fungere da serbatoio per un maggior volume di urina. In questa fase iniziale l'uretere reagisce fisiologicamente incrementando l'ampiezza e la frequenza delle sue contrazioni, ma col tempo le onde di contrazione si esauriscono, per cui l'unica forza di progressione rimane la 
Tabella 2. Risposta emodinamica trifasica all'ostruzione ureterale.

\begin{tabular}{lllll}
\hline FASE & RBF & Effetto emodinamico & GFR & Pressione tubulare \\
\hline I-2 ore & Aumentato & Vasodilatazione arteria afferente & Costante & Aumentata \\
$2-5$ ore & Diminuito & Vasocostrizione arteria efferente & Diminuito & Aumentata \\
$>24$ ore & Diminuito & Vasocostrizione arteria afferente & Diminuito & Diminuita \\
\hline
\end{tabular}

RBF: flusso ematico renale; GFR: velocità di filtrazione glomerulare.

pressione idrostatica. Nelle prime tre ore la pressione "a riposo" aumenta da 0 a $42 \mathrm{~cm} \mathrm{H}_{2} \mathrm{O}$ in associazione con un incremento di diametro di circa il $16 \%$ e con un minimo incremento di lunghezza.

\section{Fase intermedia}

L'aumento di pressione raggiunge un picco nelle prime ore per poi tornare ai valori basali. Il calo pressorio è dovuto alla riduzione della produzione di urina che dipende dalla risposta emodinamica intrarenale, che verrà di seguito descritta, ma anche dal riassorbimento veno-linfatico e, in parte, da una riduzione della tensione della parete urelaterale. La dilatazione della via escretrice persiste nonostante il calo della pressione intraluminale per effetto del persistere delle modificazioni della struttura viscoelastica della parete ureterale (proprietà isteretica). Dopo sei a otto ore la pressione intraluminale rimane invariata con la progressiva deformazione circonferenziale e longitudinale dell'uretere e un marcato incremento della tensione. Dopo otto giorni il diametro è aumentato del $170 \%$ e la lunghezza del $25 \%$. Si sviluppa inoltre una ipertrofia muscolare che implica una maggior forza contrattile circonferenziale, che è ulteriormente aumentata dal rimaneggiamento della direzione dei fasci muscolari. Tuttavia nonostante l'ipertrofia e l'aumentata forza e tensione contrattile della componente muscolare della parete ureterale non si genera una maggior pressione intraluminale. Il paradosso di una ridotta pressione intraluminale in presenza di una maggior forza contrattile è spiegata dalla legge di Laplace che lega la tensione parietale (T) al prodotto della pressione intraluminale $(\mathrm{P})$ per il raggio (r) del contenitore, cioè dell'uretere. In altre parole ciò significa che, a parità di tensione, all'aumentare del raggio diminuisce la pressione intraluminale.

\section{Fase tardiva}

Dopo 2 settimane di ostruzione sperimentale l'uretere si allunga del $24 \%$, il diametro aumenta del $100 \%$ e l'area della sezione muscolare del $248 \%$. L'uretere conserva una buona contrattilità ma non è in grado di generare valori pressori efficaci alla progressione dell'urina.

\section{Emodinamica renale}

Parallelamente alle alterazioni morfo-funzionali dell'uretere, l'ostruzione provoca modificazioni dell'emodinamica renale. Gran parte delle informazioni di cui disponiamo dipende da una lunga serie di studi sperimentali coordinati da Gillenwatere, in seguito, da Vaughan, che a Charlottesville e successivamente a New York, hanno estensivamente studiato questi aspetti fisiologici. ${ }^{15-17}$ In particolare negli anni settanta è stata descritta la risposta trifasica del flusso ematico renale (RBF) in risposta alla variazione della pressione ureterale dopo ostruzione ureterale unilaterale (tabella 2).

\section{Prima fase}

La fase iniziale, che interessa i primi 90 minuti, è caratterizzata da un aumento di RBF secondaria ad una vasodilatazione pre-glomerulare che rappresenta una risposta compensatoria intesa ad aumentare la pressione capillare e, conseguentemente, a conservare la filtrazione glomerulare (GFR).

\section{Seconda fase}

La seconda fase (da 90 minuti a 4-5 ore) è caratterizzata da un calo del RBF pur in presenza di elevati valori di pressione nella via escretrice. Si associa ad una vasocostrizione postglomerulare in un ulteriore tentativo di mantenere la GFR.

\section{Terza fase}

La terza fase ( $>5$ ore) si presenta con un calo di RBF in associazione ad un calo della pressione ureterale ed è caratterizzata ad una vasocostrizione preglomerulare. Dopo 24 ore la pressione ureterale e tubulare calano rispettivamente del 30 e $50 \%$.

\section{Mediatori bioumorali}

Per spiegare la fase iniziale è stata presa in considerazione l'aumentata escrezione di prostaglandine E2, in quanto è stato dimostrato sperimentalmente che l'indometacina provoca un immediato declino del flusso ematico renale prima dell'ostruzione ed inibisce la fase vasodilatatoria dopo ostruzione. ${ }^{18}$ Successivamente, è stata enfatizzata l'importanza dell'ossido nitrico, che viene prodotto per conversione enzimatica dell'arginina dalla ossido nitrico sintetasi (NOS) che è presente nel rene normale e la cui isoforma inducibile (iNOS) è aumentata nel rene ostruito. Infatti lo stiramento delle cellule endoteliali secondario 
all'aumento della pressione idrostatica glomerulare può provocare il rilascio di ossido nitrico. L'inibizione di NOS inibisce l'aumento di flusso renale plasmatico ${ }^{19} \mathrm{e}$ l'infusione di L-arginina contrasta l'inibizione dell'aumento di RBF mediato da indometacina. ${ }^{20}$ Studi di micropuntura hanno confermato l'aumento della pressione idrostatica dei capillari glomerulari dopo ostruzione ureterale acuta in associazione al rilascio di ossido nitrico secondario a stiramento endoteliale. ${ }^{21} \mathrm{Si}$ deve tuttavia sottolineare che molte evidenze sperimentali sono relative a studi in animali con reni uni-caliciali (ratto, cane) e non sono stati confermati in animali con via escretrice multi-calciale (maiale, agnello, babbuino). Diversi mediatori sono stati inizialmente considerati per spiegare la fase vasocostrittiva. Inizialmente è stato considerata l'ipotesi di un'attivazione del sistema renina/angiotensina II per spiegare le modificazioni emodinamiche intrarenali tardive post-ostruttive. Sono stati descritti elevati livelli di renina ed angiotensina dopo ostruzione in modelli animali (ratto e cane) e generazione intrarenale di angiotensina II (maiale). Tuttavia i risultati del trattamento farmacologico (inibitori ACE e AT blockers) in corso di ostruzione hanno dato risultati non univoci. ${ }^{22} \mathrm{I}$ trombossani (TXA2 e TXB2) sono potenti vasocostrittori, che potrebbero derivare dall'infiltrato infiammatorio renale attraverso il rilascio di fattore attivante le piastrine (platelet activating factor, PAF). Schreiner ha infatti dimostrato che la riduzione dell'infiltrato cellulare attraverso irradiazione del rene ostruito riduce l'escrezione di TXB2 con miglioramento di RBG e GFR. Tuttavia la somministrazione di inibitori della sintesi di TXA2 o il blocco dei relativi recettori non è sempre efficace nel conservare RFB e GFR. ${ }^{23}$

\section{Ostruzione bilaterale}

Pur non essendo oggetto di questo articolo, è importante ricordare che in caso di ostruzione bilaterale non si osserva la risposta trifasica. Infatti, dopo una iniziale e transitorio aumento di RBF dovuto a vasodilatazione pre-glomerulare, si osserva un marcato calo di RBF in associazione ad elevata pressione ureterale dovute a vasocostrizione postglomerulare. Viene quindi a mancare la terza fase di vasocostrizione pre-glomerulare per effetto dell'accumulo di peptide atriale natriuretico che viene rilasciato in risposta all'aumento del volume circolante con lo scopo di incrementare diuresi enatriuresi attraverso la vasodilatazione pre-glomerulare e vasocostrizione post-glomerulare, l' aumentato coefficiente di ultrafiltrazione dei capillari glomerulari e l'inibizione diretta del feed-back tubuloglomerulare (renina/angiotensina). ${ }^{24}$

\section{Risposta infiammatoria e fibrosi interstiziale}

In tempi più recenti, l'attenzione si è concentrata sullo stress ossidativo e le conseguenti risposte immunologiche che si osservano a seguito dell'ostruzione ureterale unilaterale che è così diventata un modello di studio dei meccanismi di progressione della fibrosi delle malattie renali in generale. ${ }^{23}$ Infatti già poche ore dopo l'inizio della ostruzione ureterale si innescano meccanismi di stress ossidativo, che contribuiscono significativamente alla risposta infiammatoria e alla sregolazione di fattori di crescita, fattori di trascrizione e citochine fibrogenetiche. ${ }^{25}$ L'aumento delle specie reattive dell'ossigeno (reactive oxygen species, ROS) insieme ad altri fattori intrarenali induce un ambiente renale che favorisce l'atrofia tubulare per effetto dell'apoptosi ed una progressiva fibrosi interstiziale per effetto della trasformazione delle cellule epiteliali in fibroblasti attivati. In questo processo è implicata la complessa interazione di mediatori come il fattore di crescita trasformante beta (transforming growth factor-beta, TGF- $\beta$ ) e l'ossido nitrico. Il TGF- $\beta$ è un mediatore della fibrosi e dell'apoptosi, mentre l'ossido nitrico è un mediatore multifunzionale che oltra a modificare l'emodinamica intrarenale ha dimostrato un'azione anti-apoptotica and anti-fibrotica. Già dopo 3 giorni di ostruzione unilaterale acuta dell'uretere si osserva un incremento di TGF- $\beta$, mediata dalla distensione tubulare. ${ }^{26} \mathrm{Si}$ sviluppa un marcato quadro infiammatorio con deposizione di un eccesso di matrice extracellulare secondaria all'aumentata sintesi di collagene e fibronectina per l'attivazione di fibroblasti e miofibroblasti. ${ }^{27}$ L'infiltrazione leucocitaria tubulo-interstiziale è precoce e prominente e si associa ad un aumento della popolazione macrofagica, delle chemochine e dei loro recettori e di altre molecole come osteopontina, galectina-3, selectine, fattori di crescita piastrinico (platelet-derived growth factor-D, PDGF-D) e macrofagico (macrophage-colony stimulating factor, M-CSF). Nel processo di fibrinogenesi e apoptosi sono coinvolti il sistema renina-angiotensina, il fattore nucleare kappa B (NF-kB) e le vie MAPK. ${ }^{28}$ Questi processi determinano una progressiva perdita di nefroni con peggioramento della funzionalità renale. Dopo una settimana dall'inizio dell'ostruzione sono già presenti segni di atrofia tubulare e fibrosi tubulo-interstiziale come conseguenza della risposta infiammatoria e dell'apoptosi. Se l'ostruzione è rimossa precocemente si osserva una lenta e quasi completa risoluzione della infiltrazione tubulointerstiziale con recupero del flusso renale plasmatico e del GFR, ma quando l'ostruzione si prolunga ulteriormente il processo di atrofia e fibrosi prosegue con perdita della massa tubulare prossimale e formazione di glomeruli atubulari e perdita funzionale irreversibile. Il recupero dipende dalla durata dell'ostruzione e dal modello animale in studio. Uno studio sperimentale nel topo ha dimostrato che una disostruzione precoce, dopo sette giorni, è in grado di ripristinare, a 30 giorni di distanza, la normalità di alcuni parametri anatomico-funzionali del rene ostruito come il peso dell'organo, il volume glomerulare e dell'area tubulare prossimale e la quantità di 
deposizione di collagene. ${ }^{29}$ Tuttavia a distanza di tempo è stata osservata una persistente perdita del $40 \%$ del flusso renale plasmatico e del GFR con un aumento di 2,8 volte del rapporto urinario albumina/proteine in associazione con una riduzione dell'integrità glomerulo-tubulare nel $50 \%$. In un altro modello sperimentale nel ratto, dopo un periodo di solo tre giorni di ostruzione si è osservato un recupero dei parametri funzionali dopo 14 giorni, ma con persistente incremento dei valori tissutali di TGF- $\beta 1$, in associazione a quadri di persistente fibrosi ed apoptosi. ${ }^{30}$

\section{Funzione renale postostruttiva}

Dopo la disostruzione ureterale si manifestano numerose alterazioni della funzione renale tra loro interdipendenti, che sono ben descritte nella letteratura ${ }^{31} \mathrm{ma}$ non sono oggetto di questa analisi. Di maggior interesse sono gli effetti funzionali a lungo termine di brevi periodi di ostruzione, come quelli che caratterizzano la colica renale. In generale, periodi di ostruzione superiori alle 72 ore si associano a un decremento permanente del GFR, mentre per periodi più brevi si osserva un recupero del GFR sebbene non associato ad un omogeneo recupero del GFR dei singoli nefroni (SNGFR). I nefroni più superficiali sarebbero meno sensibili agli effetti dell'ostruzione rispetto a quelli profondi. Le alterazioni della funzione tubulare tendono a persistere anche dopo che sono stati ripristinati normali valori di GFR. Dopo un'ostruzione di 24 ore, si osserva una riduzione iniziale del GFR, che torna a valori normali dopo 14 giorni, grazie all'incremento del SNGFR della popolazione nefronica residua. Il recupero del GFR è quindi attribuibile ad un'iperfiltrazione dei nefroni residui. Parallelamente la funzione tubulare rimane alterata con persistenti difetti della capacità di concentrare ed acidificare le urine e dell'escrezione di potassio, che possono essere secondaria a una perdita selettiva dei nefroni iuxtamidollari o a una alterazione della funzione tubulare di tutta la popolazione nefronica. La perdita nefronica sembra essere permanente perché si mantiene fino a 60 giorni di osservazione, analogamente ai difetti tubulari. ${ }^{32}$

\section{Trattamento della calcolosi ureterale con ostruzione ureterale}

La disponibilità di un gran numero di evidenze sperimentali in relazione agli effetti dell'ostruzione ureterale deve essere attentamente considerata nella pianificazione del trattamento della calcolosi ureterale ostruente. Infatti il ripristino di valori di normale funzionalità renale dopo il sollievo dall'ostruzione non esclude la possibilità dell'instaurarsi di un successivo e progressivo danno della funzione renale. Nella colica renale secondaria a calcolosi ureterale l'ostruzione è spesso parziale ed intermittente, tuttavia non può essere escluso che anche in queste condizioni possano instaurarsi meccanismi fisiopatologici nocivi per la funzione renale. Dobbiamo quindi riconsiderare le nostre strategie in merito alla terapia espulsiva della calcolosi ureterale ed ai provvedimenti terapeutici urologici d'urgenza in caso di ostruzione secondaria a calcolosi ureterale.

\section{Terapia espulsiva}

L'espulsione spontanea del calcolo può essere considerata la soluzione più fisiologica e meno dannosa della calcolosi ureterale in quanto qualsiasi intervento strumentale è gravato da un tasso, più o meno alto, di complicanze. D'altra parte dobbiamo anche attentamente considerare i potenziali danni dell'ostruzione ureterale prolungata e della potenziale sovrapposizione infettiva. In particolare è sempre più comune il riscontro di gravi episodi settici in pazienti con calcolosi ostruente anche di modeste dimensioni, come conseguenza della crescente diffusione delle resistenze batteriche agli antibiotici che rende più difficile il trattamento empirico domiciliare dei pazienti. ${ }^{33}$ Deve essere quindi valutata con attenzione la probabilità che il trattamento espulsivo possa essere efficace in funzione delle dimensioni del calcolo e delle caratteristiche della via escretrice, la modalità del trattamento espulsivo, del follow-up e della durata massima per cui questo trattamento può essere prolungato. Ovviamente non esiste un preciso limite dimensionale del calcolo per escludere il trattamento espulsivo, tuttavia ci sono precise evidenze che dimostrano come la probabilità di espulsione spontanea del calcolo diminuisca con l'aumentare delle dimensioni del calcolo. Per un diametro di $2-4 \mathrm{~mm}$ la probabilità di espulsione spontanea è del $76 \%$, mentre per calcoli $>9 \mathrm{~mm}$ si riduce al $25 \% .{ }^{34}$ Negli ultimi anni si è affermato un approccio meno aggressivo alla colica renale, escludendo il ricorso all'iperidratazione concentrata in un breve periodo di tempo ("colpo d'acqua") che può provocare una paresi del tratto urinario ostruito fino alla rottura dei fornici con formazione di urinomi perirenali. Si è inoltre abbandonato l'impiego degli anticolinergici a favore degli alfa-bloccanti (o dei calcio-antagonisti) che avrebbero un effetto più specifico e fisiologico sulla parete muscolare dell'uretere. L'efficacia dell'impiego degli alfa-bloccanti è stato tuttavia oggetto di discordanti valutazioni che sono state recentemente riassunte da un gruppo di lavoro dell'Associazione Europea di Urologia (EAU), che ha concluso che, sulla base delle evidenze disponibili, il trattamento espulsivo con alfabloccanti può dare un potenziale beneficio solo per calcoli dell'uretere distale $>5 \mathrm{~mm} .{ }^{35}$

Infatti una metanalisi di studi randomizzati ${ }^{36}$ ha dimostrato un maggior tasso di espulsione nei pazienti trattati con alfa-bloccanti rispetto ai controlli (RR 1,48) con un più breve tempo di espulsione $(-2,91 \mathrm{gg})$. L'uso di alfa-bloccanti riduce il numero di episodi dolorosi $(-0.48)$, la necessità di analgesici $(-38.17 \mathrm{mg})$ e di ospedalizzazione $(-0.35)$ al costo di una maggior incidenza effetti collaterali $(+2.74)$ seppur di lieve entità. 
Una seconda metanalisi ${ }^{37}$ degli studi pubblicati tra il 1990 e il 2016 ha confermato che gli alfa-bloccanti sono efficaci nell'aumentare il tasso di espulsione ( $78 \%$ vs $74 \%$ ) soprattutto nei pazienti con calcoli $>5 \mathrm{~mm}$ e dell'uretere distale, mentre i calcio antagonisti non migliorano il tasso di espulsione rispetto ai controlli ( $79 \%$ vs $75 \%$ ).

La somministrazione di corticosteroidi garantisce un piccolo effetto additivo all'azione degli alfa-bloccanti come dimostrato in uno studio randomizzato. ${ }^{38}$

Le evidenze relative alla durata massima del trattamento espulsivo del calcolo sono assai limitate. Dagli studi sperimentali precedentemente descritti potrebbe essere desunto un tempo limite di 15 giorni, sebbene questo limite derivi da studi in specie animali con via escretrice monocaliciale più esposta agli effetti dell'ostruzione rispetto a sistemi urinari più complessi pluri-caliciali come quelli di altre specie animali (pecora, maiale, primati) e dell'uomo. D'altra parte esiste una aneddotica storica che riferisce di ripresa della funzionalità renale dopo prolungati periodi di ostruzione. ${ }^{39-41}$ Tuttavia un recente studio clinico in pazienti sottoposti ad intervento disostruttivo dopo ostruzione ureterale iatrogenica ha dimostrato, in base ai valori stimati della clearance della creatinina e della scintigrafia con mercaptoacetiltriglicina (MAG), un deterioramento della funzionalità renale a lungo termine (mediana 60,8 mesi) nei pazienti in cui il sollievo dall'ostruzione era avvenuto oltre i 14 giorni. ${ }^{42}$ L'età e lo spessore ecografico del parenchima renale si sono confermati fattori prognostici del recupero di valori normali di creatininemia ${ }^{43}$ e la normalizzazione dell'indice di resistenza renale dopo disostruzione può essere un buon predittore del recupero del flusso ematico renale e dei valori di creatininemia dopo ostruzione ureterale parziale ${ }^{44,45}$ In base ai dati sperimentali ed alla limitate evidenze cliniche si può probabilmente identificare un limite clinico precauzionale di 15 giorni entro cui si può mantenere una condizione di ostruzione ureterale senza temere conseguenze sulla funzionalità renale. Ovviamente questa osservazione deve essere contemperata, in caso di calcolo ureterale, dal fatto che l'ostruzione in questo caso potrebbe essere incompleta ed incostante in funzione dello spostamento del calcolo e dell'edema della mucosa.

\section{Derivazione urinaria d'urgenza}

La derivazione urinaria d'urgenza rappresenta il primo provvedimento in caso di ostruzione ureterale acuta, in particolare quando sussistano complicazioni come una condizione di SIRS o sepsi o l'insufficienza renale acuta. La derivazione può essere conseguita attraverso un catetere (stent) transuretrale o nefrostomico. La scelta fra le due opzioni è legata alla caratteristiche del calcolo ma anche alla situazione logistica ed alla esperienza del chirurgo, in quanto non sono state riscontrate differenze in termini di efficacia nel risolvere l'ostruzione e l'infezione. ${ }^{46}$ Una meta-analisi, cha ha incluso due studi clinici randomizzati (randomized clinical trials, RTCs), ha confermato che non esiste una sostanziale superiorità per una delle due metodiche. Il tasso di complicanze è più alto dopo nefrostomia $(4 \%)$, sebbene le complicanze dopo stenting siano probabilmente sottoriportate. D'altra parte lo stenting ureterale ha un costo superiore rispetto allo nefrostomia. ${ }^{47} \mathrm{Si}$ deve inoltre ricordare che la presenza dello stent causa una dilatazione dell'uretere che provoca uno stiramento delle cellule muscolari liscie del sincizio muscolare della parete dell'uretere con disfunzione della peristalsi. La disfunzione peristaltica implica un rallentamento del flusso urinario che, insieme con il reflusso di urina attraverso lo stent privo di meccanismi valvolari, può implicare il mantenimento della idronefrosi. A questo fenomeno corrisponde a livello molecolare una progressiva riduzione dell'espressione di Gli-1 nel muscolo liscio, che regredisce dopo la rimozione dello stent. ${ }^{48}$ Infine la presenza di uno stent può implicare una sintomatologia dolorosa, più o meno grave, conseguente all'irritazione dell'urotelio a contatto con un corpo estraneo e ai movimenti dello stent nell'uretere e nella vescica.

\section{Trattamento d'urgenza della calcolosi ureterale}

Negli ultimi anni è stato proposto da numerosi centri il trattamento d'urgenza della calcolosi ureterale in occasione della prima presentazione della colica renale. Questo approccio avrebbe il vantaggio di eseguire il trattamento prima che si instauri l'edema della parete ureterale che causa il fenomeno dell" "impattamento" che può rendere meno efficace il trattamento extracorporeo e più difficoltoso il trattamento endoscopico. Una recente metaanalisi ha considerato sei studi che hanno confrontato il trattamento extracorporeo della calcolosi ureterale in condizioni di urgenza o di elezione e altri quattro studi che hanno confrontato il risultato della ureterolitotrissia urgente o differita. ${ }^{49}$ Il trattamento extracorporeo in urgenza è stato associato ad un maggior successo in termini di percentuale di pazienti liberi da calcolo (stone free) e di necessità di manovre ancillari (cateterismi ureterali, nefrostomie, ecc). Viceversa l'ureterorenoscopia in urgenza non ha conseguito risultati migliori della stessa procedura in elezione in termini di stone-free rate, complicanze e tasso di manovre ancillari.

\section{Conclusioni}

Queste evidenze sperimentali e cliniche devono far prendere in considerazione l'ipotesi che ripetuti episodi ostruttivi, anche se parziali o transitori, possano contribuire, insieme ad altre numerose possibili concause, alla maggior prevalenza di insufficienza renale terminale osservata in pazienti con calcolosi renale. ${ }^{50}$ In relazione alla elevata prevalenza della calcolosi renale questa possibilità deve essere attentamente valutata allo scopo di ottimizzare i 
percorsi diagnostico-terapeutici per la gestione dei pazienti con colica renale secondaria a calcolosi ureterale. Nei pazienti con segni suggestivi di SIRS o con sepsi urinaria o con aumento degli indici di resistività renale è raccomandabile una pronta derivazione urinaria. La nefrostomia non interferisce con la peristalsi ureterale ed il processo di espulsione del calcolo ma è gravata da una maggior morbilità ed è poco accettata dai pazienti. Lo stenting urinario è una procedura meno invasiva ma interferisce sulla motilità dell'uretere e in molti casi è poco tollerato per i disturbi minzionali e dolorosi. Il trattamento di urgenza della calcolosi ureterale rappresenta una possibile alternativa, in assenza di infezione, ma richiede una elevata logistica organizzativa. L'espulsione spontanea rappresenta un'opzione per i pazienti senza segni di infezione ed ostruzione, ma il trattamento espulsivo implica un attento e frequente monitoraggio e deve essere limitato nel tempo. Ovviamente tutte queste raccomandazioni devono trovare applicazione in un sistema sanitario con richieste limitate, per cui è auspicabile un'azione allo scopo di far conoscere e riconoscere queste problematiche cliniche da parte delle autorità sanitarie.

\section{Dichiarazione di assenza di conflitto di interessi}

Gli Autori dichiarano di non avere conflitti di interessi.

\section{Finanziamenti}

Gli Autori dichiarano di non aver ricevuto finanziamenti specifici da qualsiasi ente nei settori pubblico, privato o senza fini di lucro.

\section{Bibliografia}

1. Cervellin G, Mora R, Ticinesi A, et al. Epidemiology and outcomes of acute abdominal pain in a large urban Emergency Department: retrospective analysis of 5,340 cases. Ann Transl Med 2016; 4: 362.

2. Trinchieri A, Cappoli S, Esposito N, et al. Epidemiology of renal colic in a district general hospital. Arch Ital Urol Androl 2008; 80: 1-4.

3. Holdgate A e Pollock T. Nonsteroidal anti-inflammatory drugs (NSAIDs) versus opioids for acute renal colic. Cochrane Database Syst Rev 2005; (2): CD004137.

4. Afshar K, Jafari S, Marks AJ, et al. Nonsteroidal antiinflammatory drugs (NSAIDs) and non-opioids for acute renal colic. Cochrane Database Syst Rev 2015; (6): CD006027.

5. Pathan SA, Mitra B, Romero L, et al. What is the best analgesic option for patients presenting with renal colic to the emergency department? Protocol for a systematic review and meta-analysis. BMJ Open 2017; 7: e015002.

6. Türk C, Petřík A, Sarica K, et al. EAU guidelines on diagnosis and conservative management of Urolithiasis. Eur Urol 2016; 69: 468-474.

7. Hill WG. Control of urinary drainage and voiding. Clin J Am Soc Nephrol 2015; 10: 480-492.

8. Morales PA. Ureteral delivery and efflux. In: Boyarsky S, Gottschalk CW e Tanagho EA (eds). Urodynamics:
Hydrodynamics of the ureter and renal pelvis. New York: Academic Press, 1971, pp.87-98.

9. Rose JG e Gillenwater JY. Pathophysiology of ureteral obstruction. Am J Physiol 1973; 225: 830-837.

10. Biancani P, Zabinski MP e Weiss RM. Bidimensional deformation of acutely obstructed in vitro rabbit ureter $\mathrm{Am} \mathrm{J}$ Physiol 1973; 225: 671.

11. Biancani P, Zabinski MP e Weiss RM. Time course of ureteral changes with obstruction. Am J Physiol 1976; 231 : 393-398.

12. Hausman M, Biancani P e Weiss RM. Obstruction induced changes in longitudinal force-length relations of rabbit ureter. Invest Urol 1979; 17: 223-226.

13. Biancani P, Hausman M e Weiss RM. Effect of obstruction on ureteral circumferential force-length relations. $\mathrm{Am} \mathrm{J}$ Physiol 1982; 243: F204-F210.

14. Weiss RM e Biancani P. Rationale for ureteral tapering. Urology 1982; 20: 482-487.

15. Moody TE, Vaughan ED Jr e Gillenwater JY. Comparison of the renal hemodynamic response to unilateral and bilateral ureteral occlusion. Invest Urol 1977; 14: 455-459.

16. Vaughan ED Jr, Marion D, Poppas DP, et al. Pathophysiology of unilateral ureteral obstruction: studies from Charlottesville to New York. Urol 2004; 172: 2563-2569.

17. Felsen D, Schulsinger D, Gross SS, et al. Renal hemodynamic and ureteral pressure changes in response to ureteral obstruction: the role of nitric oxide. J Urol 2003; 169: 373-376.

18. Allen JT, Vaughan ED Jr e Gillenwater JY. The effect of indomethacin on renal blood flow and uretral pressure in unilateral ureteral obstruction in a awake dogs. Invest Urol 1978; 15: 324-327.

19. Lanzone JA, Gulmi FA, Chou SY, et al. Renal hemodynamics in acute unilateral ureteral obstruction: contribution of endothelium-derived relaxing factor. J Urol 1995; 153: 2055-2059.

20. Schulsinger DA, Gulmi FA, Chou SY, et al. Activation of endothelium-derived relaxing factor system in acute unilateral ureteral obstruction. J Urol 1997; 157: 19511956.

21. Kramer HJ, Horacek V, Bäcker A, et al. Relative roles of nitric oxide, prostanoids and angiotensin II in the regulation of canine glomerular hemodynamics. A micropuncture study. Kidney Blood Press Res 2004; 27: 10-17.

22. Moody TE, Vaughan ED Jr, Wyker AT, et al. The role of intrarenal angiotensin II in the hemodynamic response to unilateral obstructive uropathy. Invest Urol 1977; 14: 390-397.

23. Schreiner GF, Harris KP, Purkerson ML, et al. Immunological aspects of acute ureteral obstruction: immune cell infiltrate in the kidney. Kidney Int 1988; 34: 487-493.

24. Cogan MG Renal effects of atrial natriuretic factor. Annu Rev Physiol 1990; 52: 699-708.

25. Dendooven A, Ishola DA Jr, Nguyen TQ, et al. Oxidative stress in obstructive nephropathy. Int J Exp Pathol 2011; 92: 202-210.

26. Wright EJ, McCaffrey TA, Robertson AP, et al. Chronic unilateral ureteral obstruction is associated with interstitial fibrosis and tubular expression of transforming growth factor-beta. Lab Invest 1996; 74: 528-537. 
27. Klahr S e Morrissey J. Obstructive nephropathy and renal fibrosis. Am J Physiol Renal Physiol 2002; 283: F861-F875.

28. Chevalier RL, Forbes MS e Thornhill BA. Ureteral obstruction as a model of renal interstitial fibrosis and obstructive nephropathy. Kidney Int 2009; 75: 1145-1152.

29. Chaabane W, Praddaude F, Buleon M, et al. Renal functional decline and glomerulotubular injury are arrested but not restored by release of unilateral ureteral obstruction (UUO). Am J Physiol Renal Physiol 2013; 304: F432-F439.

30. Ito K, Chen J, El Chaar M, et al. Renal damage progresses despite improvement of renal function after relief of unilateral ureteral obstruction in adult rats. Am J Physiol Renal Physiol 2004; 287: F1283-F1293.

31. Wilson DR. Pathophysiology of obstructive nephropathy. Kidney Int 1980; 18: 281-292.

32. Bander SJ, Buerkert JE, Martin D, et al. Long-term effects of 24-hr unilateral ureteral obstruction on renal function in the rat. Kidney Int 1985; 28: 614-620.

33. Reyner K, Heffner AC e Karvetski CH. Urinary obstruction is an important complicating factor in patients with septic shock due to urinary infection. Am J Emerg Med 2016; 34: 694-696.

34. Coll DM, Varanelli MJ e Smith RC. Relationship of spontaneous passage of ureteral calculi to stone size and location as revealed by unenhanced helical CT. AJR Am J Roentgenol 2002; 178: 101-103.

35. Turk C, Knoll T, Seitz C, et al. Medical expulsive therapy for ureterolithiasis: the EAU recommendations in 2016. Eur Urol 2017; 71: 504-507.

36. Campschroer T, Zhu Y, Duijvesz D, et al. Alpha-blockers as medical expulsive therapy for ureteral stones. Cochrane Database Syst Rev 2014; (4): CD008509.

37. Amer T, Osman B, Johnstone A, et al. Medical expulsive therapy for ureteric stones: Analysing the evidence from systematic reviews and meta-analysis of powered doubleblinded randomised controlled trials. Arab J Urol 2017; 15: 83-93.

38. Dellabella M, Milanese Ge Muzzonigro G. Medical-expulsive therapy for distal ureterolithiasis: randomized prospective study on role of corticosteroids used in combination with tamsulosin-simplified treatment regimen and health-related quality of life. Urology 2005; 66: 712-715.
39. Okubo K, Suzuki Y, Ishitoya S, et al. Recovery of renal function after 153 days of complete unilateral ureteral obstruction J Urol 1998; 160: 1422-1423.

40. Dhabuwala $\mathrm{CB}$ e Pearlmutter AD. Recovery of renal function in a child after prolonged, complete ureteral obstruction J Urol 1982; 127: 756-757.

41. Shapiro SR e Bennet AH. Recovery of renal function after prolonged unilateral ureteral obstruction. J Urol 1976; 115: 136-140.

42. Lucarelli G, Ditonno P, Bettocchi C, et al. Delayed relief of ureteral obstruction is implicated in the long-term development of renal damage and arterial hypertension in patients with unilateral ureteral injury. $J$ Urol 2013; 189: 960-965.

43. Lutaif NA, Yu L e Abdulkader RC. Factors influencing the non-recovery of renal function after the relief of urinary tract obstruction in women with cancer of cervix. Ren Fail 2003; 25: 215-223.

44. Shokeir AA, Nijman RJ, el-Azab M, et al. Partial ureteral obstruction: role of renal resistive index in stages of obstruction and release. Urology 1997; 49: 528-535.

45. Khalaf IM, Shokeir AA, El-Gyoushi FI, et al. Recoverability of renal function after treatment of adult patients with unilateral obstructive uropathy and normal contralateral kidney: a prospective study. Urology 2004; 64: 664-668.

46. Pearle MS, Pierce HL, Miller GL, et al. Optimal method of urgent decompression of the collecting system for obstruction and infection due to ureteral calculi. J Urol 1998; 160: 1260-1264.

47. Ramsey S, Robertson A, Ablett MJ, et al. Evidence-based drainage of infected hydronephrosis secondary to ureteric calculi. J Endourol 2010; 24: 185-189.

48. Janssen C, Buttyan R, Seow CY, et al. A role for the hedgehog effector Gli-1 in mediating stent-induced ureteral smooth muscle dysfunction and aperistalsis. Urology 2017; 104: 242e1-242e8.

49. Arcaniolo D, De Sio M, Rassweiler J, et al. Emergent versus delayed lithotripsy for obstructing ureteral stones: a cumulative analysis of comparative studies. Urolithiasis 2017; 45: 563-572.

50. Rule AD, Bergstralh EJ, Melton LJ 3rd, et al. Kidney stones and the risk for chronic kidney disease. Clin J Am Soc Nephrol 2009; 4: 804-811. 